\title{
BROWNIAN MOTION WITH INERT DRIFT, BUT WITHOUT FLUX: A MODEL
}

\section{Krzysztof Burdzy ${ }^{1}$, Robert Hołyst ${ }^{2}$ and Lukasz Pruski $^{3}$}

${ }^{1}$ Department of Mathematics, Box 354350, University of Washington, Seattle, WA 98115-4350, USA

2 Institute of Physical Chemistry PAS, Dept.III, Kasprzaka 44/52, 01224 Warsaw, Poland and WMP-SNŚ UKSW, Dewajtis 5, Warsaw, Poland

${ }^{3}$ Department of Mathematics and Computer Science, University of San Diego, 5998 Alcala Park, San Diego, CA 92110-2492, USA

\begin{abstract}
We study the motion of a Brownian particle which interacts with a stationary obstacle in two dimensions. The Brownian particle acquires drift proportionally to the time spent on the boundary of the obstacle. The system approaches equilibrium, and the equilibrium distribution for the location and drift magnitude has the product form. The distribution for the location is uniform, while the drift distribution depends on the shape of the obstacle, resembling a gamma function for the circular or elliptic obstacle.
\end{abstract}

\section{INTRODUCTION}

The Brownian motion in confined geometry can change the diffusion coefficient[1-3]. In particular, the diffusion coefficient may depend on the local position with respect to the bounding surfaces. It was shown experimentally that a Brownian particle moving in a wedge shape geometry acquires drift along the wedge axis whose magnitude is proportional to the gradient of the diffusion coefficient[4]. The gradient arises from the proximity of the walls bounding the system. Despite the fact that the displacement of a particle grows linearly with time the particle's distribution is stationary and no concentration gradients are observable. It means that despite the existence of the drift there are no fluxes in the system i.e., the system is in thermodynamic equilibrium. The lack of concentration gradients means that the drift velocity is not induced by the external field. In short, the system exhibits "a drift without flux" and drift is induced by the bounding walls.

The purpose of this paper is to present a simple yet mathematically tractable model of "a Brownian motion with drift, but without flux." In our model a Brownian particle 
interacts with a stationary obstacle. At each encounter it acquires a drift perpendicular to the obstacle. The total drift changes in the course of time as it is proportional to the number of times a particle hits the boundary. The main objective of the current study is to find the long-time behavior of Brownian motion with drift in multidimensional domains with boundary. In other words, we are interested in the stationary or equilibrium distributions of the position and drift. At the moment we do not have analytical (mathematical) tools to study this problem so our results are based on computer simulations. We will show that such a system has an equilibrium distribution of drift and location i.e., without fluxes. Moreover, the distribution of the particle position is uniform and the drift distribution is independent from particle's position with respect to the boundary.

The motivation for this model is also provided by complex systems with memory. Some aspects of the behavior of such a system may be modelled by a diffusion process with an interaction with the boundary. A simple example may be an animal or group of animals confined to an ecological niche[5]. The population dynamics of the animals may be mostly random but there may be some drift representing the memory of the past. Another, perhaps more interesting, example may be some quantity related to financial markets. If the government has a history of intervention, for example, via adjustment of interest rates, this might be sometimes modelled as an interaction with a boundary. The memory of past interactions with the boundary may add a drift to otherwise balanced diffusive behavior of a market indicator.

\section{THE MODEL}

We will present some results on a model originally proposed in [6] and developed in $[7,8,9]$. The model has two mathematically equivalent representations in one dimension. We start with the original one-dimensional model introduced in [6]. Consider two stochastic processes $Z(t)$ and $Y(t)$. The dynamics of the system $(Z(t), Y(t))$ is the following. Assume that $Z(0)>Y(0)$. In absence of interactions between the particles, the first particle $Z(t)$ is moving at a constant speed, and the second one $Y(t)$ is moving as a Brownian motion $B(t)$. Assume that the initial speed of $Z(t)$ is 0 . When the particles meet, they reflect from each other instantaneously. Let $L(t)$ be the number of times they meet before $t$. In the continuous limit, $L(t)$ is known as the local time (see [10] or [6]). The velocity $V(t)$ of $Z(t)$ increases proportionally to $L(t)$, that is, $V(t)=c_{1} L(t)$, but $Z(t)$ is not instantly

pushed at the moment of interaction. In other words, $Z(t)=\int_{0}^{t} c_{1} L(s) d s$. The effect of interactions on the second particle is that of a shift, that is $Y(t)=B(t)-c_{2} L(t)$, where $B(t)$ is a Brownian motion. After a finite random time $T$, the velocity of $Z(t)$ will increase 
to the point that $Z(t)$ will escape to infinity with a constant speed and $Y(t)$ will never hit $Z(t)$ after time $T$. The construction of the process, proof of its existence, and finiteness of time $T$ can be found in $[6,7,8]$.

We will now describe another model, mathematically equivalent to the first one. Recall that $Z(t)=\int_{0}^{t} c_{1} L(s) d s$. We subtract $\int_{0}^{t} c_{1} L(s) d s$ from both components of $(Z(t), Y(t))$ so that we obtain $(0, X(t))=(0, Y(t)-Z(t))$. In other words, the total information about the dynamics of the two-particle system is now encoded in the dynamics of a single process $X(t)$. This process has the representation $X(t)=B(t)-c_{2} L(t)-\int_{0}^{t} c_{1} L(s) d s$. The process $X(t)$ is confined to $(-\infty, 0]$. Now $L(t)$ represents the number of hits of 0 by $X(t)$, that is, the local time spent by $X(t)$ at 0 , the boundary of the fixed domain $(-\infty, 0]$.

Our paper is concerned with the two-dimensional version of the model. We chose the second mathematical representation of the one-dimensional model as the basis for the multidimensional model. In dimensions 2 and higher, the process $X(t)$ moves in a multidimensional domain $D$ that has a piecewise smooth boundary $A$. The process $X(t)$ moves like a Brownian motion $B(t)$ with a constant drift $K(t)$ when it is away from $A$. Let $N(x)$ be the unit inward normal vector to $A$ at $x \in A$ and let $L(t)$ be the number of collisions of $X(t)$ with the boundary $A$ by the time $t$. When the process hits $A$ at time $s$, two things happen. First, the process instantly reflects along the normal inner vector, that is,

$$
X(s+d s)=X(s)+c_{2} N(X(s)) \frac{d}{d s} L(s) d s,
$$

where $c_{2}>0$ is a constant chosen so that the process always stays within the domain $D$. Second, the drift $K(t)$ is modified as follows,

$$
K(s+d s)=K(s)+c_{1} N(X(s)) \frac{d}{d s} L(s) d s
$$

where $c_{1}>0$ is a constant expressing the strength of the memory of the interaction, as expressed by the inert drift. Overall, the process $X(t)$ has the following representation,

$$
X(t)=B(t)+\int_{0}^{t} c_{2} N(X(s)) \frac{d}{d s} L(s) d s+\int_{0}^{t} K(s) d s
$$

where

$$
K(t)=\int_{0}^{t} c_{1} N(X(s)) \frac{d}{d s} L(s) d s .
$$

The existence of this process has been proved in $[7,8]$. 


\section{RESULTS}

Simulations of multidimensional Brownian motion are notoriously slow so we performed simulations only in two dimensions. In order to be able to understand the overall effect of the interactions with the boundary, we chose three simple boundary shapes. In each case, the simulation box was a two-dimensional square of size 800 by 800 with periodic boundary conditions (the torus). The shapes of the obstacles in the middle of the system were a disc, an ellipse and a square (Fig.1). The details of numerical simulations are the following. In each case, a single run consisted of $10^{9}$ steps. Multiple runs were performed. Since the results were consistent across all runs, we present the results for one run for different obstacles.

Away from the boundary of an obstacle, a single step of the simulation was the superposition of the Brownian motion and the drift, as represented by $\frac{d}{d t} B(t) d t+K(t) d t$. We used the value $c_{1}=0.1$ in formula (2) for the drift because it gave the most meaningful results. The value of $c_{2}$ in (1) was determined implicitly by the fact that the collisions affected only the Brownian particle - it had to stay outside the obstacle. On the boundary, i.e., during a collision, only the normal component of the drift changed, by the value $c_{1} N(X(t))$. The tangential component of the drift remained unchanged during a collision.

In the first domain, the reflecting boundary was a circle with radius 150 . We recorded statistics for the position and drift magnitude uniformly over the whole time interval, and also for two other cases. In the first case, we recorded drift magnitude statistics for the times when the particle was close to the obstacle, that is, within $10 \%$ of the maximum distance from the reflecting circle. In the other case, we recorded drift magnitude statistics for the times when the particle was far from the obstacle, that is, farther than $70 \%$ of the maximum distance from the circle.

In the second domain, the obstacle had the shape of an ellipse with semi-axes of length 150 and 100. We recorded statistics for the position and drift magnitude uniformly over the whole time interval, and also for two other cases. In the first case, we recorded drift magnitude statistics for the times when the particle was close to the obstacle. In the other case, we recorded drift magnitude statistics for the times when the particle was far from the obstacle. The part of the domain "close" to the ellipse was obtained as the region of the original domain within the ellipse dilated by the factor of 1.45. The part of the domain "far" from the ellipse was obtained as the region of the original domain outside the ellipse dilated by the factor of 3.37. The "close" range comprised about $9 \%$ of all locations that the particle occupied during the simulation. The "far" range contained about $28 \%$ of all 
particle locations.

In the third domain, the obstacle was a square with side length 150 . We recorded statistics for the position and drift magnitude uniformly over the whole time interval, and also for two other cases. In the first case, we recorded drift magnitude statistics for the times when the particle was close to the obstacle, that is, within $10 \%$ of the maximum distance from the square. In the other case, we recorded drift magnitude statistics for the times when the particle was far from the obstacle, that is, farther than $70 \%$ of the maximum distance from the square.

Our findings are the following. The stationary distribution for the particle is uniform in the domain where it can move. We verified this in three ways. First, the density graph for the particle distribution is visually very close to the uniform distribution. Second, we compared statistics for the distance of the particle from the obstacle with the same statistics for the (theoretical) uniform distribution. Finally, we divided the domain into 6400 boxes and calculated the ratios of the actual frequencies in the boxes and the theoretical frequencies based on the uniform distribution. We calculated the standard deviation for the 6400 ratios and concluded that the simulation data supported the claim of uniformity of the distribution. Each of the three procedures described above strongly indicated that the stationary distribution was uniform.

Our second finding is that the joint stationary distribution for the position of the particle and its drift is the product of the stationary distribution for the position and the stationary distribution for the drift. In other words, the stationary distribution for the two observables has the product form. The numerical evidence for this assertion was generated as follows. In each of the domains, we determined the drift magnitude profile for three particle position subdomains. In other words, we determined the overall drift magnitude profile, then we determined the drift magnitude profile when the particle position was close to the edge of the obstacle, and finally we determined the drift magnitude profile when the particle position was far from the edge of the obstacle. All three profiles are the same, in each of the domains that we considered. The discrete nature of the simulations shows in the data on the drift magnitude in the domain with the square obstacle (Fig. 7). Nevertheless, even in this case there is an excellent agreement between the three drift magnitude profiles.

The distribution of the drift magnitude has a unimodal shape. The shape of the distribution resembles that of a gamma distribution but the best fit of a gamma distribution based on matching moments (mean and variance) is not quite satisfactory. 


\section{SUMMARY}

It is known that the stationary distribution for the Brownian motion with normal reflection on the boundary of the domain and no drift is uniform in the domain. This can be derived from the Markovian nature of Brownian motion and time-reversibility of the Brownian motion with the normal reflection vector. We note that in general, the stationary distribution is not uniform, if the reflection on the boundary is not normal (see [11]). In our model, the pair of processes $(X(t), K(t))$ has the Markov property and is time-reversible. However, the particle position $X(t)$ alone is not a Markov process and one cannot apply the theory of Markov processes to $X(t)$ alone to find its stationary distribution. We have shown using numerical simulations that in the long time limit, Brownian motion with drift acquired at the boundary approaches the uniform spatial distribution and drift distribution independent from the spatial distribution. The surprising find is that the interaction of the particle with the boundary of the domain in the form of the inert drift does not affect the stationary distribution for particle position.

Acknowledgements

Research partially supported by NSF grant DMS-0600206 (KB and LP) and as a scientific project during 2006-2008 from the budget of Ministry of Science and Higher Education $(\mathrm{RH})$.

\section{REFERENCES}

1 H.Brenner, J.Fluid Mech. 12 (1962) 35.

2 H.Brenner Chem. Eng. Sci. 16 (1961) 242

3 L.P. Faucheux, A.J. Libchaber, Phys.Rev. E 49 (1994) 5158.

4 P.Lancon, G.Batrouni, L.Lobry and N.Ostrowsky, Physica A 304 (2002) 65.

5 S.T.Lindley, Ecological Applications, 13 (2003) 806.

6 F. Knight, On the path of an inert object impinged on one side by a Brownian particle. Probab. Theory Related Fields 121, (2001) 577-598.

7 D. White, Processes with inert drift. Ph.D. Thesis, University of Washington (2005)

8 D. White, Processes with inert drift. Mathematics ArXiv, preprint math.PR/0604052 (2006)

9 K. Burdzy and D. White, A Gaussian oscillator Electron. Comm. Probab. 9 (2004) paper 10, pp. 92-95.

10 I. Karatzas and S. Shreve Brownian Motion and Stochastic Calculus, Second Edition. Springer-Verlag, New York, 1994. 
11 J.M. Harrison, H.J. Landau and L.A. Shepp, The stationary distribution of reflected Brownian motion in a planar region. Ann. Probab. 13 (1985), 744-757.

\section{FIGURE CAPTIONS}

Figure 1. The simulation box with periodic boundary conditions. An obstacle is located inside the box. The three obstacles studied were: a disc, an ellipse and a square. A Brownian particle acquires drift at the boundary of the obstacle. A Brownian particle cannot penetrate the obstacle. The size of the system is 800 by 800 .

Figure 2. The stationary distribution of the distance of the particle from the edge of the disc (filled circles) and the distribution of the distance of uniformly chosen point in the domain from the edge of the disc (empty circles). The size of the simulation box is 800 by 800 . The horizontal axis represents the distance from the obstacle. The vertical axis represents the observed relative frequency. The area under the graph represents the total probability (100\%). There were about 400 data points. They were combined into 40 bins.

Figure 3. Drift magnitude profiles in a domain with disc-shaped obstacle. Empty circles - data for all positions of the particle. Filled circles - data for particle position closer than $10 \%$ of the maximum distance to the disc edge. Squares - data for particle position farther than $70 \%$ of the maximum distance from the disk edge. The size of the simulation box is 800 by 800 . The horizontal axis represents the drift magnitude. The vertical axis represents the observed relative frequency. The area under the graph represents the total probability (100\%). The solid line represents the density of the gamma distribution with the mean and variance matching those of the data for all positions of the particle (mean $=0.3434$, standard deviation $=0.1503$ ). There were about 400 data points. They were combined into 40 bins.

Figure 4. Elliptic obstacle. The stationary distribution of the distance of the particle from the center of the ellipse (filled circles) and the distribution of the distance of uniformly chosen point in the domain from the center of the ellipse (empty circles). The size of the simulation box is 800 by 800 . The horizontal axis represents the distance from the center of the obstacle. The vertical axis represents the observed relative frequency. The area under the graph represents the total probability (100\%). There were about 
450 data points. They were combined into 40 bins.

Figure 5. Drift magnitude profiles in a domain with an elliptic obstacle. Empty circles - data for all positions of the particle. Filled circles - data for particle position close to the edge of the ellipse. Squares - data for particle position far from the ellipse edge. The size of the simulation box is 800 by 800 . The horizontal axis represents the drift magnitude. The vertical axis represents the observed relative frequency. The area under the graph represents the total probability $(100 \%)$. The solid line represents the density of the gamma distribution with the mean and variance matching those of the data for all positions of the particle $($ mean $=0.3372$, standard deviation $=0.1498)$. There were about 400 data points. They were combined into 40 bins.

Figure 6. Square obstacle. The stationary distribution of the distance of the particle from the edge of the square (filled circles) and the distribution of the distance of uniformly chosen point in the domain from the edge of the square (empty circles). The size of the simulation box is 800 by 800 . The horizontal axis represents the distance from the obstacle. The vertical axis represents the observed relative frequency. The area under the graph represents the total probability (100\%). There were about 450 data points. They were combined into 40 bins.

Figure 7. Profiles of the logarithm of the drift magnitude in a domain with a square obstacle. Filled circles - data for all positions of the particle. Circles - data for particle position closer than $10 \%$ of the maximum distance to the square edge. Squares - data for particle position farther than $70 \%$ of the maximum distance to the square edge. The size of the simulation box is 800 by 800 . The horizontal axis represents the drift magnitude. The vertical axis represents the observed relative frequency. The area under the graph represents the total probability (100\%). The number of distinct observed drift magnitude values was 32 . 

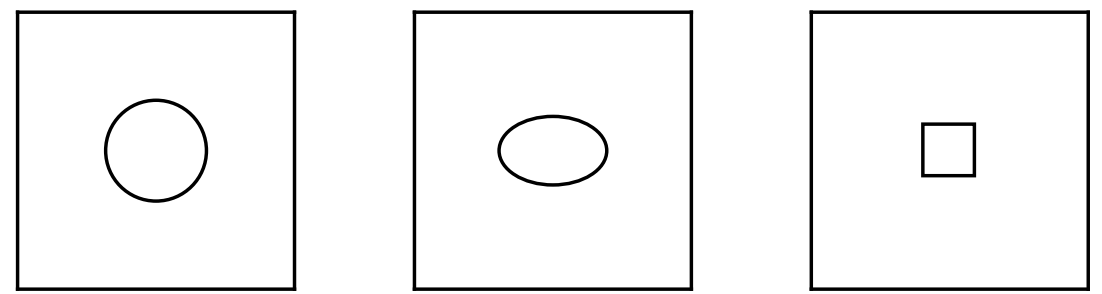

Figure 1. The simulation box with periodic boundary conditions. An obstacle is located inside the box. The three obstacles studied were: a disc, an ellipse and a square. A Brownian particle acquires drift at the boundary of the obstacle. A Brownian particle cannot penetrate the obstacle. The size of the system is 800 by 800 . 


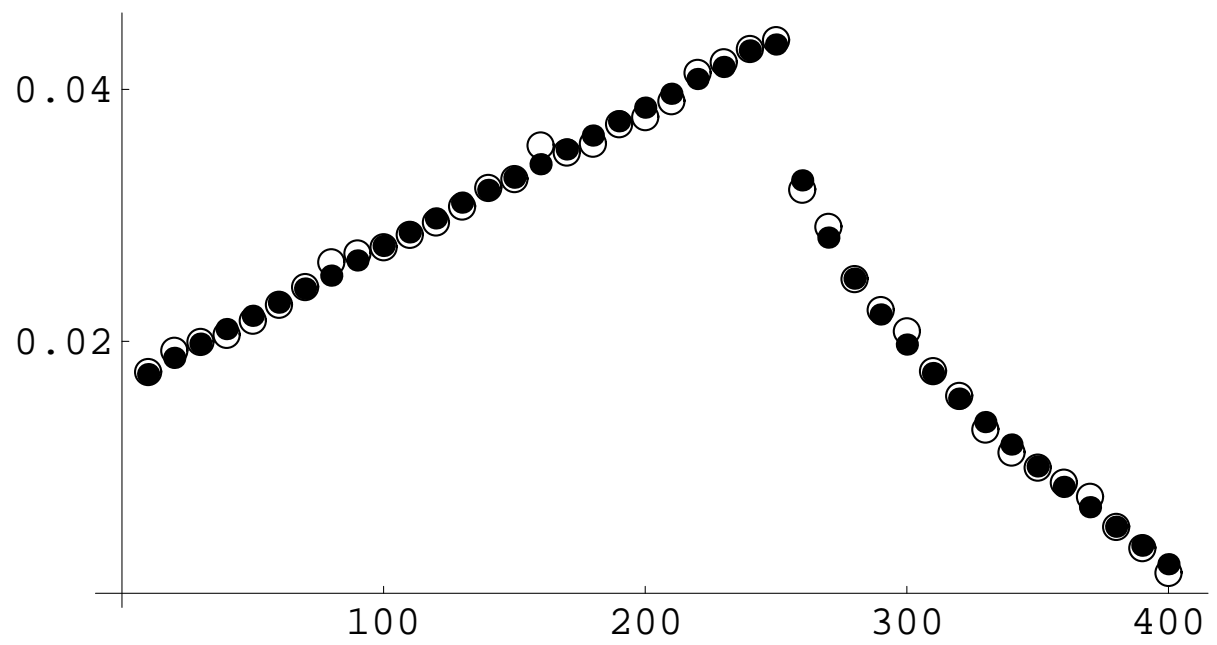

Figure 2. The stationary distribution of the distance of the particle from the edge of the disc (filled circles) and the distribution of the distance of uniformly chosen point in the domain from the edge of the disc (circles). The size of the simulation box is 800 by 800 . The horizontal axis represents the distance from the obstacle. The vertical axis represents the observed relative frequency. The area under the graph represents the total probability (100\%). There were about 400 data points. They were combined into 40 bins. 


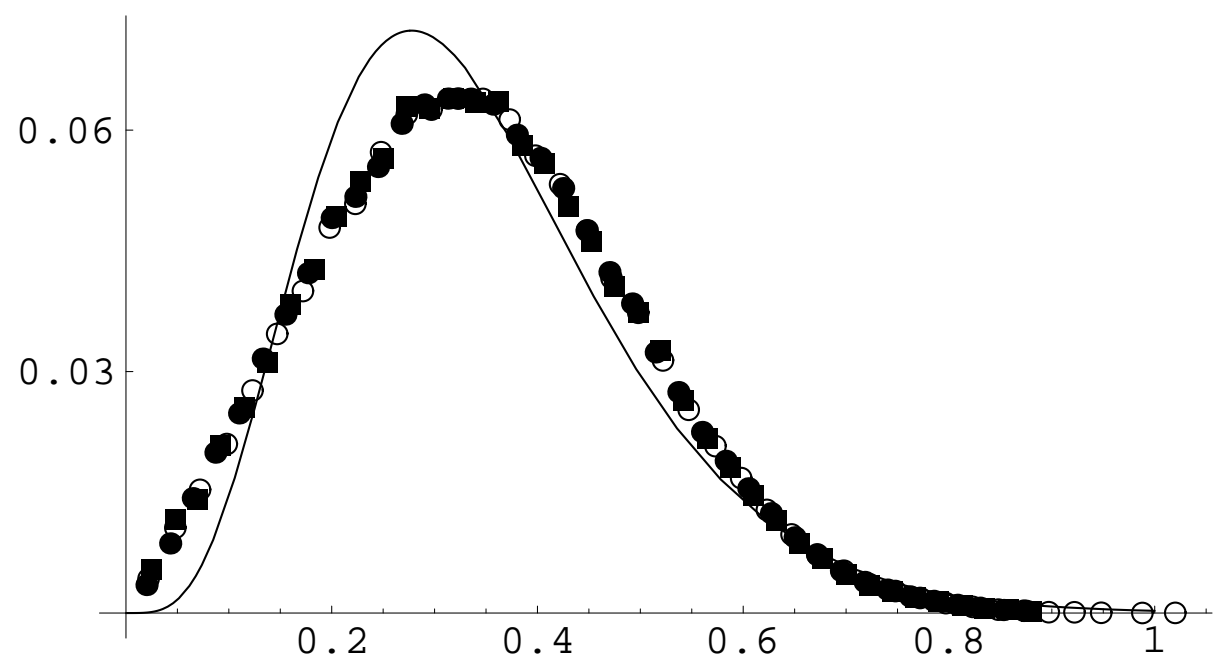

Figure 3. Drift magnitude profiles in a domain with disc-shaped obstacle. Empty circles - data for all positions of the particle. Filled circles - data for particle position closer than $10 \%$ of the maximum distance to the disc edge. Squares - data for particle position farther than $70 \%$ of the maximum distance from the disk edge. The size of the simulation box is 800 by 800 . The horizontal axis represents the drift magnitude. The vertical axis represents the observed relative frequency. The area under the graph represents the total probability (100\%). The solid line represents the density of the gamma distribution with the mean and variance matching those of the data for all positions of the particle (mean $=0.3434$, standard deviation $=0.1503)$. There were about 400 data points. They were combined into 40 bins. 


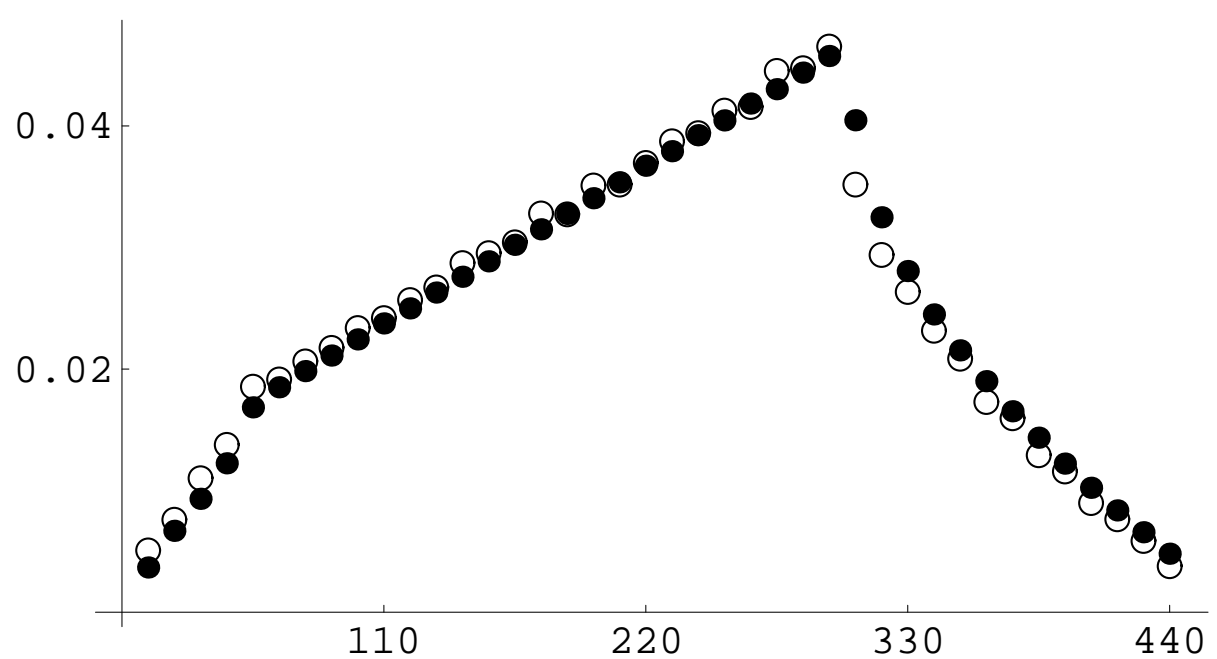

Figure 4. Elliptic obstacle. The stationary distribution of the distance of the particle from the center of the ellipse (filled circles) and the distribution of the distance of uniformly chosen point in the domain from the center of the ellipse (circles). The size of the simulation box is 800 by 800 . The horizontal axis represents the distance from the center of the obstacle. The vertical axis represents the observed relative frequency. The area under the graph represents the total probability (100\%). There were about 450 data points. They were combined into 40 bins. 


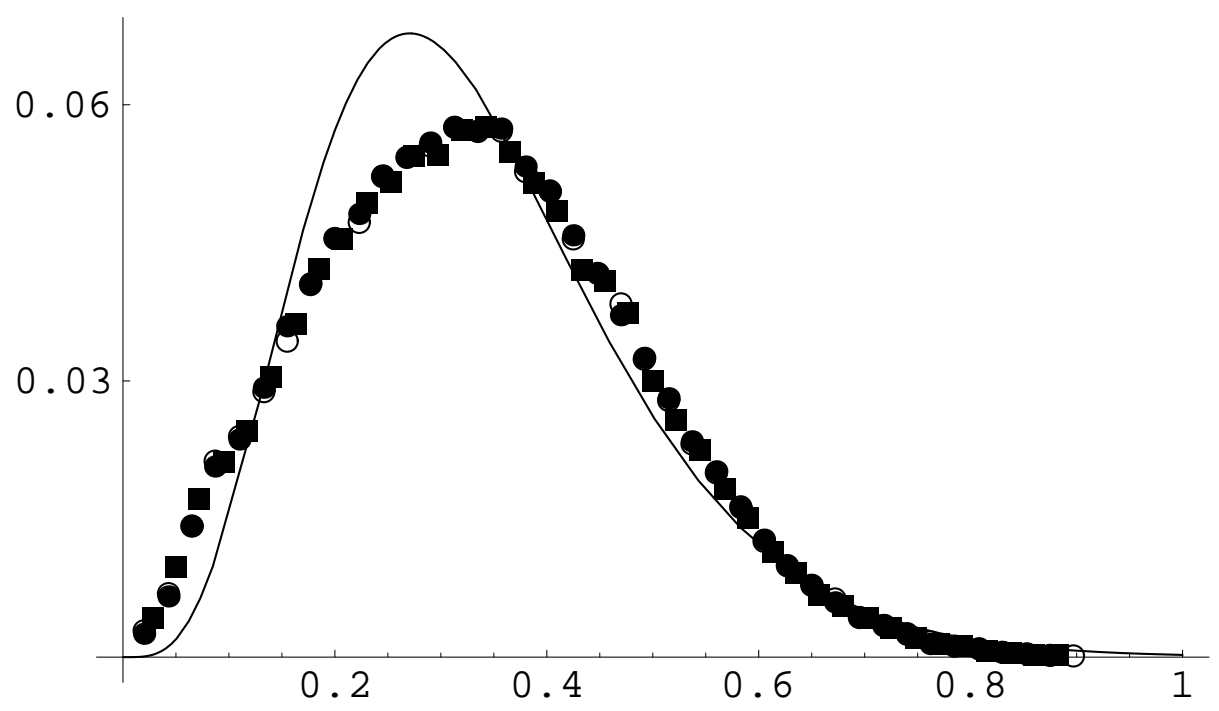

Figure 5. Drift magnitude profiles in a domain with an elliptic obstacle. Empty circles - data for all positions of the particle. Filled circles - data for particle position close to the edge of the ellipse. Squares - data for particle position far from the ellipse edge. The size of the simulation box is 800 by 800 . The horizontal axis represents the drift magnitude. The vertical axis represents the observed relative frequency. The area under the graph represents the total probability (100\%). The solid line represents the density of the gamma distribution with the mean and variance matching those of the data for all positions of the particle $($ mean $=0.3372$, standard deviation $=0.1498$ ). There were about 400 data points. They were combined into 40 bins. 


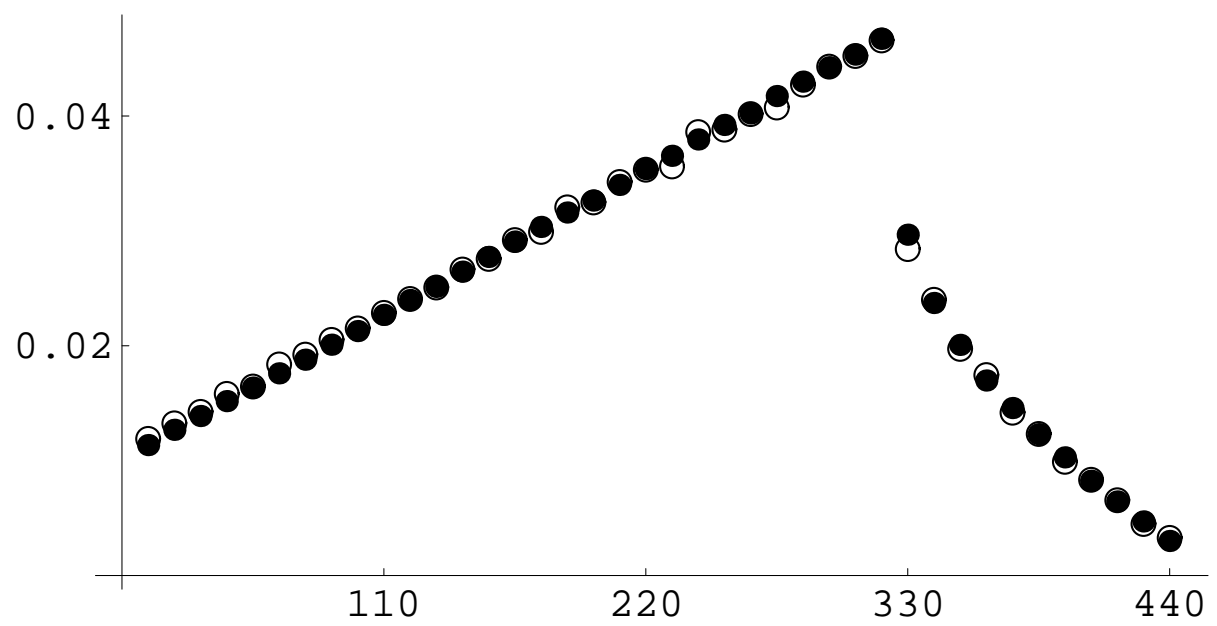

Figure 6. Square obstacle. The stationary distribution of the distance of the particle from the edge of the square (filled circles) and the distribution of the distance of uniformly chosen point in the domain from the edge of the square (empty circles). The size of the simulation box is 800 by 800 . The horizontal axis represents the distance from the obstacle. The vertical axis represents the observed relative frequency. The area under the graph represents the total probability (100\%). There were about 450 data points. They were combined into 40 bins. 


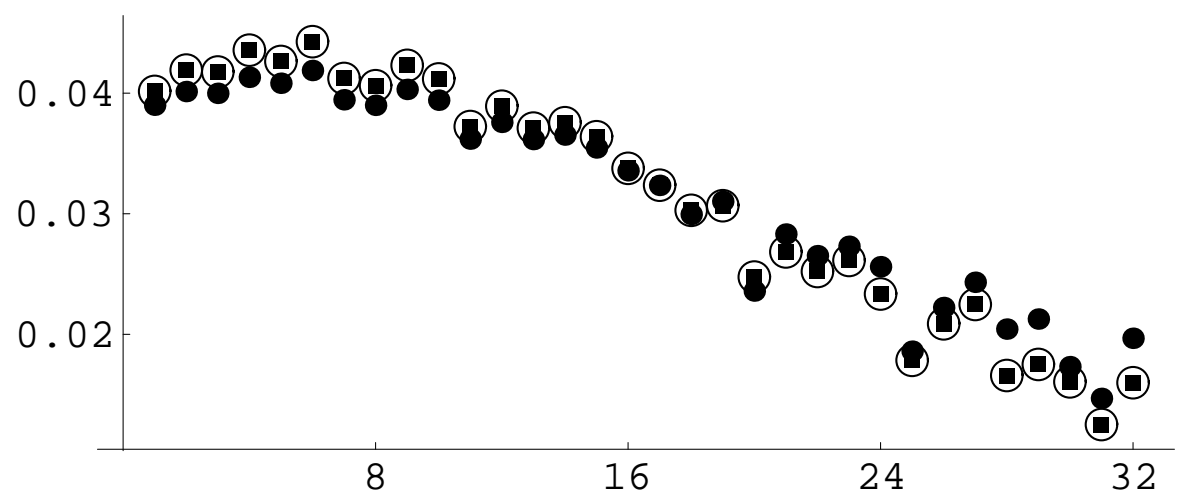

Figure 7. Profiles of the logarithm of the drift magnitude in a domain with a square obstacle. Filled circles - data for all positions of the particle. Circles - data for particle position closer than $10 \%$ of the maximum distance to the square edge. Squares - data for particle position farther than $70 \%$ of the maximum distance to the square edge. The size of the simulation box is 800 by 800 . The horizontal axis represents the drift magnitude. The vertical axis represents the observed relative frequency. The area under the graph represents the total probability $(100 \%)$. The number of distinct observed drift magnitude values was 32 . 